Y. Teranishi

Nagoya Math. J.

Vol. 104 (1986), 149-161

\title{
THE RING OF INVARIANTS OF MATRICES
}

\author{
YASUO TERANISHI
}

\section{§1. Introduction}

We denote by $M(n)$ the space of all $n \times n$-matrices with their coefficients in the complex number field $C$ and by $G$ the group of invertible matrices $G L(n, C)$. Let $W=M(n)^{l}$ be the vector space of $l$-tuples of $n \times$ $n$-matrices. We denote by $\rho: G \rightarrow G L(W)$ a rational representation of $G$ defined as follows:

$$
\rho(S)(A(1), A(2), \cdots, A(l))=\left(S A(1) S^{-1}, S A(2) S^{-1}, \cdots, S A(l) S^{-1}\right)
$$

if $S \in G, A(i) \in M(n)(i=1,2, \cdots, l)$.

This action of $G$ defines an action of $G$ on an algebra $C[W]=C\left[x_{i j}(1)\right.$, $\left.\cdots, x_{i j}(l)\right]$ of all polynomial functions on $W$. We denote by $C[W]^{G}$ the subalgebra of $G$ invariant polynomials. This is a finitely generated subalgebra of $C[W]$.

If $l=1$ it is a classical result that this ring of invariants is a polynomial ring in $n$ variables. In fact the coefficients of characteristic polynomial of the matrix $X(1)=\left(x_{i j}(1)\right)$ are algebraically independent invariants and the ring of invariants is generated by them. By the Newton's formula all coefficients of characteristic polynomial of $X(1)$ are expressed by $n$ traces

$$
\operatorname{Tr}(X(1)), \operatorname{Tr}\left(X^{2}(1)\right), \cdots, \operatorname{Tr}\left(X(1)^{n}\right),
$$

and hence $C\left[x_{i j}(1)\right]^{G}$ is the polynomial ring generated by these traces.

Procesi [5] has shown the following important

THEOREM 1.1. The ring of invariants $C[W]^{G}$ is generated by all traces $\operatorname{Tr}\left(X\left(i_{1}\right) \cdots X\left(i_{j}\right)\right)(j=1,2, \cdots)$, where $X\left(i_{1}\right) \cdots X\left(i_{j}\right)$ runs all possible noncommutative monomials.

The object of this paper is to determine the Poincare series of $C[W]^{G}$ and to determine generators of $C[W]^{G}$ for some cases.

Received June 13, 1985. 
The following notations are fixed throughout:

$C$ the field of complex numbers

$N$ additive semigroup of nonnegative integers

$\boldsymbol{Q}$ the field of rational numbers

For a complex number $z$, we denote by $\bar{z}$ its complex conjugate and set $e(z)=\exp 2 \pi \sqrt{-1} z$.

The author expresses his hearty thanks to Dr. Daniele Montanari who pointed out a mistake in the earlier version of this paper.

\section{$\S 2$. Poincaré series}

We give $C[W]$ the structure of $N^{l}$-graded algebra by defining deg $x_{i j}(k)$ to be the $k$-th unit coordinate vector $\varepsilon_{k}$ in $N^{l}$. Let

$$
C[W]=\bigoplus_{d \in N^{l}} C[W]_{d},
$$

where $C[W]_{d}$ is a vector space spanned over $C$ by the monomials in $C[W]$ of degree $d \in N^{l}$. Then $C[W]^{G}$ has the structure

$$
C[W]^{G}=\underset{d \in N^{l}}{\oplus} C[W]_{d}^{G},
$$

of an $N^{l}$-graded algebra given by

$$
C[W]_{d}^{G}=C[W]^{G} \cap C[W]_{d} .
$$

The Poincare series of $C[W]^{G}$ is the formal power series $P\left(z_{1}, \cdots, z_{l}\right)$ in $l$-variables $z_{1}, \cdots, z_{l}$ defined by

$$
P\left(z_{1}, \cdots, z_{l}\right)=\sum_{d \in N^{l}} \operatorname{dim}_{C} C[W]_{d}^{G} z^{d}
$$

where $z^{d}=z_{1}^{d_{1}} \cdots z_{l}^{d_{l}}$ with $d=\left(d_{1}, \cdots, d_{l}\right)$.

A theorem of Hilbert-Serre implies that $P\left(z_{1}, \cdots, z_{l}\right)$ is a rational function in $l$ variables $z_{1}, \cdots, z_{l}$. By using a classical method of Molien-Weyl, we shall calculate this rational function.

For each diagonal unitary matrix $\varepsilon$ with diagonal entries

$$
\varepsilon_{1}, \varepsilon_{2}, \cdots, \varepsilon_{n}
$$

since $\left|\varepsilon_{i}\right|=1(i=1,2, \cdots, n)$, we can put $\varepsilon_{i}=e\left(\varphi_{i}\right)\left(0 \leqq \varphi_{i} \leqq 1\right)$. We set

$$
\Delta=\prod_{i<j}\left(e\left(\varphi_{i}\right)-e\left(\varphi_{j}\right)\right) \text {. }
$$

Then the normalized volume element on the group consisting of diagonal unitary matrices is given by 


$$
\frac{1}{n !} \Delta \bar{J} d \varphi_{1} \cdots d \varphi_{n},[8]
$$

We define polynomials in one variable $z$ by

$$
\Delta(z)=\prod_{i<. l}\left(e\left(\varphi_{i}\right)-z e\left(\varphi_{j}\right)\right)
$$

and

$$
\bar{\Delta}(z)=\prod_{i<1}\left(e\left(\varphi_{i}\right)-z \overline{e\left(\varphi_{\jmath}\right)}\right) .
$$

Theorem 2.1. The Poincaré series $P\left(z_{1}, \cdots, z_{l}\right)$ is

$$
\begin{gathered}
\frac{1}{n ! \prod_{i=1}^{l}\left(1-z_{i}\right)^{n}} \int_{0}^{1} \cdots \int_{0}^{1} \frac{\Delta \bar{J}}{\prod_{l=1}^{l} \Delta\left(\boldsymbol{z}_{i}\right) \bar{J}\left(z_{i}\right)} d \varphi_{1} \cdots d \varphi_{n}, \\
\left|z_{1}\right|<1, \cdots,\left|z_{l}\right|<1 .
\end{gathered}
$$

Proof. Let $f(z)$ be a polynomial in one variable $z$ defined as

$$
\begin{aligned}
f(z) & =\operatorname{det}\left(I_{n}-\rho(\varepsilon) z\right), \quad I_{n}=\text { the } n \times n \text {-identity matrix, } \\
& =\prod_{1 \leqq \imath<j \leqq n}\left(1-z \varepsilon_{i} \varepsilon_{j}^{-1}\right) \\
& =(1-z)^{n} \Delta(z) \bar{J}(z) .
\end{aligned}
$$

Then by the Molien-Weyl formula [8], the Poincaré series $P\left(z_{1}, \cdots, z_{l}\right)$ equals

$$
\frac{1}{n !} \int_{0}^{1} \cdots \int_{0}^{1} \frac{\Delta \bar{\Delta}}{f\left(z_{1}\right) \cdots f\left(z_{l}\right)} d \varphi_{1} \cdots d \varphi_{n}, \quad\left|z_{i}\right|<1
$$

By changing variables from $\varphi_{1}, \cdots, \varphi_{n}$ to $\varepsilon_{1}, \cdots, \varepsilon_{n}$, we have

$$
\begin{aligned}
& P\left(z_{1}, \cdots, z_{l}\right) \\
& \quad=\left(\frac{1}{2 \pi \sqrt{-1}}\right)^{n} \frac{1}{n ! \prod_{i=1}^{l}\left(1-z_{i}\right)^{n}} \int_{c_{1}} \cdots \int_{c_{n}} \frac{\Delta J}{\prod_{i=1}^{l} \frac{\Delta J\left(z_{i}\right) J\left(z_{i}\right)}{s_{1}} \cdots d \xi_{\xi_{i}},}
\end{aligned}
$$

where $C_{k}$ denotes the unit circle $\left|\varepsilon_{h}\right|=1$ in the complex $\varepsilon_{h}$-plane. Thus the Poincaré series $P\left(z_{1}, \cdots, z_{l}\right)$ can be calculated in principle by means of residues. Since

$$
\Delta(z) \bar{\Delta}(z)=(-z)^{(n(n-1)) / 2}\left(\varepsilon_{1} \cdots \varepsilon_{n}\right)^{1-n} \prod_{i<i}\left(\varepsilon_{i}-z \varepsilon_{j}\right)\left(\varepsilon_{\imath}-\frac{1}{z} \varepsilon_{j}\right),
$$

we have

$$
\begin{aligned}
\prod_{i=1}^{l} \frac{\Delta \bar{\Delta}}{\Delta\left(\boldsymbol{z}_{i}\right) \bar{\Delta}\left(\boldsymbol{z}_{i}\right)=}= & (-1)^{(n(n-1)(l-1)) / 2}\left(z_{1} \cdots \boldsymbol{z}_{l}\right)^{(n(1-n)) / 2}\left(\varepsilon_{1} \cdots \varepsilon_{l}\right)^{(n-1)(l-1)} \\
& \times \prod_{p=1}^{l} \frac{D\left(\varepsilon_{1}, \cdots, \varepsilon_{n}\right)}{\prod_{i<j}\left(\varepsilon_{i}-\boldsymbol{z}_{p} \varepsilon_{j}\right)\left(\varepsilon_{i}-\left(1 / \boldsymbol{z}_{p}\right) \varepsilon_{j}\right)},
\end{aligned}
$$


where $D\left(\varepsilon_{1}, \cdots, \varepsilon_{n}\right)=\prod_{i<j}\left(\varepsilon_{i}-\varepsilon_{j}\right)^{2}$. And so we can rewrite Theorem 2.1 as

$$
\begin{aligned}
& P\left(z_{1}, \cdots, z_{l}\right) \\
& =(-1)^{(n(n-1)(l-1)) / 2} \frac{1}{n ! \prod_{i=1}^{l}\left(1-z_{i}\right)^{n}\left(z_{1} \cdots z_{l}\right)^{(n(n-1)) / 2}}\left(\frac{1}{2 \pi \sqrt{-1}}\right)^{n} \\
& \quad \times \int \cdots \int \frac{\left(\varepsilon_{1} \cdots \varepsilon_{n}\right)^{(n-1)(l-1)-1} D\left(\varepsilon_{1}, \cdots, \varepsilon_{n}\right)}{\prod_{p=1}^{l} \prod_{i<j}\left(\varepsilon_{i}-z_{p} \varepsilon_{j}\right)\left(\varepsilon_{i}-\left(1 / z_{p} \varepsilon_{j}\right)\right.} d \varepsilon_{1} \cdots d \varepsilon_{n} .
\end{aligned}
$$

Proposition 2.3. The Poincaré series $P\left(z_{1}, \cdots, z_{l}\right)(l \geqq 2)$ satisfies the following functional equation

$$
P\left(z_{1}^{-1}, \cdots, z_{l}^{-1}\right)=(-1)^{n(l-1)+1}\left(z_{1}, \cdots, z_{l}\right)^{n 2} P\left(z_{1}, \cdots, z_{l}\right) .
$$

Proof. Consider a rational function $I\left(z_{1}, \cdots, z_{l}\right)$ defined in $\left|z_{1}\right|<1, \cdots$, $\left|z_{l}\right|<1$ as follows

$$
I\left(z_{1}, \cdots, z_{l}\right)=\int_{c_{1}} \cdots \int_{c_{n}} F_{z_{1}, \cdots, z_{l}}\left(\varepsilon_{1}, \cdots, \varepsilon_{n}\right) d \varepsilon_{1} \cdots d \varepsilon_{n},
$$

where

$$
F_{z_{1}, \cdots, z_{l}}\left(\varepsilon_{1}, \cdots, \varepsilon_{n}\right)=\frac{\left(\varepsilon_{1} \cdots \varepsilon_{n}\right)^{(n-1)(l-1)-1} D\left(\varepsilon_{1}, \cdots, \varepsilon_{n}\right)}{\prod_{p=1}^{l} \prod_{i<j}\left(\varepsilon_{i}-z_{p} \varepsilon_{j}\right)\left(\varepsilon_{i}-\left(1 / z_{p}\right) \varepsilon_{j}\right)} .
$$

Set inductively

$$
\begin{gathered}
I_{1}\left(\varepsilon_{1}, \cdots, \varepsilon_{n}\right)=F_{z_{1}, \cdots, z_{l}}\left(\varepsilon_{1}, \cdots, \varepsilon_{n}\right), \\
I_{i+1}\left(\varepsilon_{i+1}, \cdots, \varepsilon_{n}\right)=\int_{\left|\varepsilon_{i}\right|=1} I_{i}\left(\varepsilon_{i}, \varepsilon_{i+1}, \cdots, \varepsilon_{n}\right) d \varepsilon_{i}, \\
(i=1, \cdots, n-1) .
\end{gathered}
$$

Then we find that $I_{i}\left(\varepsilon_{i}, \cdots, \varepsilon_{n}\right)$ is, as a function of $\varepsilon_{i}$, holomorphic at $\varepsilon_{i}=\infty$. If $\left|z_{1}\right|>1, \cdots,\left|z_{l}\right|>1$, we have

$$
\begin{aligned}
I\left(z_{1}^{-1}, \cdots, z_{l}^{-1}\right) & =\int_{c_{1}} \cdots \int_{c_{n}} F_{z_{1}, \cdots, z_{l}}\left(\varepsilon_{1}, \cdots, \varepsilon_{n}\right) d \varepsilon_{1} \cdots d \varepsilon_{n} \\
& =(-1)^{n-1} \int_{c_{1}} \cdots \int_{c_{n-1}} \int_{c_{n}} F_{z_{1}}, \cdots, z_{l}\left(\varepsilon_{1}, \cdots, \varepsilon_{n}\right) d \varepsilon_{1} \cdots d \varepsilon_{n} .
\end{aligned}
$$

By the Cauchy integral formula we have

$$
I\left(z_{1}^{-1}, \cdots, z_{l}^{-1}\right)=(-1)^{n-1} I\left(z_{1}, \cdots, z_{l}\right),
$$

and hence we obtain the result by 2.2 .

We consider $C[W]$ as a $N$-graded algebra

$$
C[W]=\bigoplus_{d \in N} C[W]_{d}
$$


by defining deg $x_{i j}(k)=1$ and define the Poincaré series $P(z)$ in one variable $z$ by

$$
P(z)=P(z, \cdots, z)=\sum_{d \in N} \operatorname{dim}_{C} C[W]_{d}^{G} z^{d} .
$$

Then it follows from (2.2) that the Poincaré series $P(z)$ equals

$$
\begin{aligned}
& (-1)^{(n(n-1)(l-1)) / 2} \frac{1}{n !(1-z)^{n l} z^{(n(n-1) l) / 2}}\left(\frac{1}{2 \pi \sqrt{-1}}\right)^{n} \\
& \quad \times \int \cdots \int \frac{\left(\varepsilon_{1} \cdots \varepsilon_{n}\right)^{(n-1)(l-1)-1} D\left(\varepsilon_{1}, \cdots, \varepsilon_{n}\right)}{\left(\prod_{i<j}\left(\varepsilon_{i}-z \varepsilon_{j}\right)\left(\varepsilon_{i}-(1 / z) \varepsilon_{j}\right)\right)^{l}} d \varepsilon_{1} \cdots d \varepsilon_{n} .
\end{aligned}
$$

Let $f_{1}, \cdots, f_{m}$ be a homogeneous system of parameters of the $N$-graded algebra $C[W]^{G}$. By a theorem of Hochster and Roberts [4], $C[W]^{G}$ is a free module over the polynomial ring $C\left[f_{1}, \cdots, f_{m}\right]$. Let $\varphi_{1}, \cdots, \varphi_{r}$ be a homogeneous system of generators of this module,

$$
C[W]^{G}=\bigoplus_{i=1}^{r} \varphi_{i} C\left[f_{1}, \cdots, f_{m}\right] .
$$

We claim that $m=(l-1) n^{2}+1$. For $w \in W$, we denote by $G_{w}$ the isotropy subgroup of $G L(n, C)$ at $w$. If $l \geqq 2$, there exists a dense open subset $U$ of $w$ such that $G_{w}=\{e\}$. Then it follows from a theorem of Rosenlicht [6] that the transcendence degree of $C[W]^{G}$ is equals $\operatorname{dim} W-\operatorname{dim} G+1$. This shows that $m=(l-1) n^{2}+1$. Formanek [1] has shown that the field of rational invariants $C(W)^{G}$ is unirational of transcendence degree $(l-1) n^{2}+1$.

We set

$$
\begin{aligned}
& \operatorname{deg} f_{i}=d_{i}, \quad d_{1} \leqq \cdots \leqq d_{m} \\
& \operatorname{deg} \varphi_{j}=e_{j}, \quad 0=e_{1} \leqq \cdots \leqq e_{r} .
\end{aligned}
$$

By Proposition 2.3, $P(z)$ satisfies the following functional equation

$$
P\left(z^{-1}\right)=(-1)^{(l-1) n^{2+1}} z^{n^{2} l} P(z) .
$$

This equation is equivalent to

$$
d_{1}+\cdots+d_{m}-e_{-i+1}=n^{2} l+e_{i}, \quad i=1, \cdots, r .
$$

In particular we have

$$
\begin{aligned}
& e_{i}+e_{r-i+1}=e_{r}, \quad i=1, \cdots, l, \\
& e_{r}=d_{1}+\cdots+d_{m}-n^{2} l
\end{aligned}
$$

and 


$$
n^{2} l=\sum_{j=1}^{m} d_{j}-\frac{2}{r} \sum_{i=1}^{r} e_{i}
$$

Let $\alpha$ and $\beta$ be the first and second Laurant coefficients of $P(z)$ respectively. Then the Laurant expansion of the Poincaré series $P(z)$ begins with

$$
P(z)=\frac{\alpha}{(1-z)^{m}}+\frac{\beta}{(1-z)^{m-1}}+\cdots .
$$

By 2.5.9 Lemma (7), it follows that

$$
\alpha=\frac{r}{d_{1} \cdots d_{m}}
$$

and

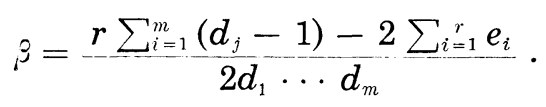

Then it follows from (2.5) that

$$
\frac{\beta}{\alpha}=\frac{n^{2}-1}{2}
$$

We shall need the following important theorem due to Hilbert [3].

THEOREM 2.8. Assume that some invariants $I_{1}, \cdots, I_{\mu}$ have a property that their vanishing implies the vanishing of all invariants. Then the ring of invariants is integral over the subring generated by $I_{1}, \cdots, I_{\mu}$.

\section{$\S 3$. The ring of invariants of $2 \times 2$ matrices}

In this section we shall be concerned with the ring of invariants of $2 \times 2$ matrices. Throughout this section we assume that $l \geqq 2$.

Proposition 3.1. (1) The Poincaré series $P_{2}(z)$ is given by

$$
P_{2}(z)=\left.(-1)^{l-1} \frac{1}{2(l-1) !(1-z)^{2 l}}\left(\frac{d}{d \varepsilon}\right)^{l-1} \frac{\varepsilon^{l-2}(\varepsilon-1)^{2}}{(z \varepsilon-1)^{l}}\right|_{\varepsilon=z} .
$$

(2) The Laurant expansion of $P_{2}(z)$ at $a=1$ begins with

$$
P_{2}(z)=\frac{[l-1]_{l-2}}{(l-1) ! 2^{2 l-1}(1-z)^{4 l-3}}+\frac{3[l-1]_{l-2}}{(l-1) ! 2^{2 l}(1-z)^{4 l-4}}+\cdots,
$$

where $[l-1]_{l-2}=(l-1) l(l+1) \cdots(2 l-4)$. 
(3) If $C[X(1), \cdots, X(l)]^{G L(2)}=\oplus_{i=1}^{r} \varphi_{i} C\left[f_{1}, \cdots, f_{4 l-3}\right]$, where $f_{1}, \cdots, f_{4 l-3}$ is a system of parameters of $C[X(1), \cdots, X(l)]^{G L(2)}$, we have

$$
r=\frac{[l-1]_{l-2}}{(l-1) !} \prod_{i=1}^{4 l-3} \frac{\operatorname{deg}\left(f_{i}\right)}{2^{2 l-1}}
$$

Proof. (1) follows from (2.4). By a direct computation, we see that the first Laurant coefficient at $z=1$ equals

$$
\begin{gathered}
{[l-1]_{l-2}} \\
(l-1) ! 2^{2 l-1}
\end{gathered}
$$

Then (2) follows from (2.7). (3) is an immediate consequence from (2) and (2.6).

We denote by $C_{l}$ a subring of $C[X(1), \cdots, X(l)]^{G L(2)}$ generated by traces $\operatorname{Tr}(X(i) X(j)), 1 \leqq i, j \leqq l, \operatorname{Tr}(X(i)), 1 \leqq i \leqq l$.

Proposition 3.2. The ring of invariants $C[X(1), \cdots, X(l)]^{G L(2)}$ is integral over $C_{l}$.

Proof. By Theorem 1.1, it is enough to show

$$
\begin{aligned}
& \text { if } \operatorname{Tr}\left(A_{i} A_{j}\right)=\operatorname{Tr}\left(A_{i}\right)=0\left(A_{i}, A_{j} \in M(2, C), 1 \leqq i, j \leqq l\right), \\
& \operatorname{Tr}\left(A_{i 1} A_{i 2} \cdots A_{\imath k}\right)=0 \quad \text { for any } k, 1 \leqq i_{1}, \cdots, i_{k} \leqq l .
\end{aligned}
$$

We shall prove $(*)$ by induction on $l$. By making the substitution $A_{i} \rightarrow$ $B A_{i} B^{-1}(B \in G L(2, C))$, we can assume $A_{1}=0$ or $A_{1}=\left(\begin{array}{ll}0 & 1 \\ 0 & 0\end{array}\right)$.

If $A_{1}=0$, by the inductive hypothesis (*) is true. If $A_{1}=\left(\begin{array}{ll}0 & 1 \\ 0 & 0\end{array}\right)$, we have $A_{i}=\left(\begin{array}{cc}0 & \alpha_{i} \\ 0 & 0\end{array}\right), a_{\imath} \in C(1 \leqq i \leqq l)$. Because $\operatorname{Tr}\left(A_{1} A_{\imath}\right)=0$ and $A_{\imath}^{2}=0$. $1 \leqq i \leqq l$. This shows that $\operatorname{Tr}\left(A_{i 1} A_{i 2} \cdots A_{i k}\right)=0$. This completes the proof.

If $l=2$ or $3, \operatorname{Tr}(X(i) X(j))(1 \leqq i, j \leqq l), \operatorname{Tr}(X(i))(1 \leqq i \leqq l)$ is a homogeneous system of parameters of $C[X(1), \cdots, X(l)]^{G L(2)}$.

Proposition 3.3. (E. Formanek, P. Halpin and W.C.W. Li [2])

$$
\begin{aligned}
& C[X(1), X(2)]^{G L(2)} \\
& \quad=C\left[\operatorname{Tr}(X(1)), \operatorname{Tr}(X(2)), \operatorname{Tr}\left(X(1)^{2}\right), \operatorname{Tr}\left(X(2)^{2}\right), \operatorname{Tr}(X(1) X(2))\right]
\end{aligned}
$$

Proof. By (3) Proposition 3.1, we have $r=1$ and we obtain the result. 


\section{$\S 4$. The ring of invariants $C[X(1), X(2)]^{G L(3)}$}

In this section we treat the case: $n=3$ and $l=2$. Set

$$
\begin{aligned}
& f_{1}=\operatorname{Tr}(X(1)), \quad f_{2}=\operatorname{Tr}\left(X(1)^{2}\right), \quad f_{3}=\operatorname{Tr}\left(X(1)^{3}\right), \\
& f_{4}=\operatorname{Tr}(X(2)), \quad f_{5}=\operatorname{Tr}\left(X(2)^{2}\right), \quad f_{6}=\operatorname{Tr}\left(X(2)^{3}\right), \\
& f_{7}=\operatorname{Tr}(X(1) X(2)), \quad f_{8}=\operatorname{Tr}\left(X(1) X(2)^{2}\right), \quad f_{9}=\operatorname{Tr}\left(X(1)^{2} X(2)\right), \\
& f_{10}=\operatorname{Tr}\left(X(1)^{2} X(2)^{2}\right), \quad f_{11}=\operatorname{Tr}\left(X(1) X(2) X(1)^{2} X(2)^{2}\right) .
\end{aligned}
$$

We denote by $C$ the subring of $C[X(1), X(2)]^{G L(3)}$ generated by ten invariants $f_{1}, \cdots, f_{10}$ which are algebraically independent.

TheOREM 4.1. $f_{1}, \cdots, f_{10}$ is a system of parameters of the ring $C[X(1)$, $X(2)]^{G L(3)}$ and

$$
C[X(1), X(2)]^{G L(3)}=C \oplus f_{11} C .
$$

Proof. Let $A_{1}$ and $A_{2}$ be $3 \times 3$-matrices which satisfy the following condition: $f_{1}\left(A_{1}, A_{2}\right)=\cdots=f_{10}\left(A_{1}, A_{2}\right)=0$.

Since $\operatorname{Tr}\left(A_{i}\right)=\operatorname{Tr}\left(A_{i}^{2}\right)=\operatorname{Tr}\left(A_{i}^{3}\right)=0, \quad i=1,2$, we have $A_{1}^{3}=A_{2}^{3}=0$. If $A_{1}^{2}=A_{2}^{2}=0$, it follows from the Cayley-Hamilton theorem that $A_{1} A_{2} A_{1}$ $=A_{2} A_{1} A_{2}=0$ and hence we have, for any $k, \operatorname{Tr}\left(A_{i_{1}} A_{i_{2}} \cdots A_{i_{k}}\right)=0,1 \leqq$ $i_{1}, \cdots, i_{k} \leqq 2$. Assume now that $A_{1}^{2} \neq 0$. Then, by making the substitution

$$
A_{i} \longrightarrow B A_{i} B^{-1}, \quad i=1,2,
$$

we can assume that $A_{1}$ and $A_{2}$ are of the form

$$
A_{1}=\left(\begin{array}{rrr}
0 & 1 & \\
& 0 & 1 \\
& & 0
\end{array}\right), \quad A_{2}=\left(\begin{array}{lll}
a_{11} & a_{12} & a_{13} \\
a_{21} & a_{22} & a_{23} \\
a_{31} & a_{32} & a_{33}
\end{array}\right) \text {. }
$$

The equations $\operatorname{Tr}\left(A_{1} A_{2}\right)=\operatorname{Tr}\left(A_{1}^{2} A_{2}\right)=\operatorname{Tr}\left(A_{2}\right)=0$ imply $a_{11}+a_{22}+a_{33}=$ $a_{21}+a_{32}=a_{31}=0$ and $\operatorname{Tr}\left(A_{1}^{2} A_{2}^{2}\right)=0$ implies $a_{21} a_{32}=0$. Hence we have $a_{31}=$ $a_{21}=a_{32}=0$. This shows that $A_{2}$ is an upper triangular matrix with zero diagonal entries. Consequently $\operatorname{Tr}\left(A_{i 1} A_{i 2} \cdots A_{i k}\right)=0, i_{1}, i_{2}, \cdots, i_{k}=1,2$ for any $k$.

If $A_{1}$ or $A_{2}$ is the zero matrix, all traces are zero by our assumption. Therefore $C[X(1), X(2)]^{G L(3)}$ is integral over $C$. Since the transcendence degree of the ring $C[X(1), X(2)]^{G L(3)}$ is ten, $f_{1}, \cdots, f_{10}$ is a homogeneous system of parameters.

Consider the Poincaré series $P\left(z_{1}, z_{2}\right)$. By the theorem of Hochster and Roberts $C[X(1), X(2)]^{G L(3)}$ is a free module over the subring $C$. Therefore 
there is a polynomial $F\left(z_{1}, z_{2}\right)$ in two variables such that

$$
\begin{aligned}
& P\left(z_{1}, z_{2}\right) \\
& =\frac{F\left(z_{1}, z_{2}\right)}{\left(1-z_{1}\right)\left(1-z_{1}^{2}\right)\left(1-z_{1}^{3}\right)\left(1-z_{2}\right)\left(1-z_{2}^{2}\right)\left(1-z_{2}^{3}\right)\left(1-z_{1} z_{2}\right)\left(1-z_{1}^{2} z_{2}\right)\left(1-z_{1} z_{2}^{2}\right)\left(1-z_{1}^{2} z_{2}^{2}\right)} .
\end{aligned}
$$

It follows from the functional equation of $P\left(z_{1}, z_{2}\right)$ that $F\left(z_{1}, z_{2}\right)$ satisfies the following relation

$$
F\left(z_{1}, z_{2}\right)=\left(z_{1} z_{2}\right)^{3} F\left(z_{1}^{-1}, z_{2}^{-1}\right) .
$$

And it is easily shown that $F\left(z_{1}, z_{2}\right)=1+z_{1}^{3} z_{2}^{3}$. Therefore $C[X(1), X(2)]^{G L(3)}$ is generated by $f_{1}, \cdots, f_{10}$ and an invariant $\varphi$ of degree $(3,3)$.

Invariants $\operatorname{Tr}\left(X(1) X(2) X(1)^{2} X(2)^{2}\right), \operatorname{Tr}\left(X(2) X(1) X(2)^{2} X(1)^{2}\right)$ and $\operatorname{Tr}(X(1)$. $X(2) X(1) X(2) X(1) X(2))$ span the vector space $C[X(1), X(2)]_{(3,3)}^{G L(3)}$ consisting of invariants of degree $(3,3)$. By the Cayley-Hamilton theorem, we find that $\operatorname{Tr}(X(1) X(2) X(1) X(2) X(1) X(2)) \in C$ and $\operatorname{Tr}\left(X(1) X(2) X(1) X(2)^{2}\right)+\operatorname{Tr}(X(2)$. $\left.X(1) X(2)^{2} X(1)^{2}\right) \in C$. Therefore the ring of invariants $C[X(1), X(2)]^{G L(3)}$ is generated by $f_{1}, \cdots, f_{11}$ and $C[X(1), X(2)]^{G L(3)}=C \oplus f_{11} C$. This completes the proof.

\section{$\S 5$. The ring of invariants $C[X(1), X(2)]^{G L(4)}$}

We denote by $\operatorname{Sym}(n)$ the symmetric group of $n$ letters and recall the multi-linearlized Cayley-Hamilton theorem for $n \times n$-matrices $Y_{1}, \cdots, Y_{n}$ :

$$
\begin{aligned}
& \sum_{\pi \in \mathrm{SYm}(n)} Y_{\pi(1)} \cdots Y_{\pi(n)} \\
& \quad+\sum_{k=1}^{n} \sum_{u} \sum_{\pi \in \mathrm{Sym}(n)} q_{u} \operatorname{Tr}\left(Y_{\pi(1)} \cdots Y_{\pi(u 1)}\right) \cdots Y_{\pi(n-k+1)} Y_{\pi(n-k+2)} \cdots Y_{\pi(n)}=0,
\end{aligned}
$$

for suitable $q_{u} \in \boldsymbol{Q}$ and suitable $j$-tuples $u=\left(u_{1}, \cdots, u_{j}\right)$ such that $1 \leqq u_{1}$ $\leqq u_{2} \leqq \cdots \leqq u_{j}$ and $u_{1}+\cdots+u_{i}=k$.

Proposition 5.1. The ring of invariants $C[X(1), X(2)]^{G L(4)}$ is generated by invariants of the form

$$
\begin{aligned}
& \operatorname{Tr}\left(X(1)^{\alpha_{1}} X(2)^{\alpha_{2}} X(1)^{\alpha_{3}} X(2)^{\alpha_{4}}\right), \quad 0 \leqq \alpha_{1}, \alpha_{2}, \alpha_{3} \leqq 3, \\
& \operatorname{Tr}\left(X(1) X(2) X(1)^{2} X(2)^{2} X(1)^{3} X(2)^{3}\right), \quad \operatorname{Tr}\left(X(1) X(2) X(1) X(2)^{2} X(1) X(2)^{3}\right), \\
& \operatorname{Tr}\left(X(2) X(1) X(2) X(1)^{2} X(2) X(1)^{3}\right) .
\end{aligned}
$$

Proof. We claim that any invariant $\operatorname{Tr}\left(X(1)^{\alpha_{1}} X(2)^{\alpha_{2}} \cdots X(1)^{\alpha_{2 r-1}} X(2)^{\alpha_{2} r}\right)$, $0 \leqq \alpha_{1}, \cdots, \alpha_{2 r} \leqq 3(r>6)$, can be written as a polynomial in $T\left(X(1)^{\beta_{1}} X(2)^{\beta_{2}}\right.$ $\cdots X(1)^{\beta_{5}} X(2)^{\beta_{6}}, 0 \leqq \beta_{1}, \cdots, \beta_{6} \leqq 3$. We work by induction on $r$. We assume 
that, for any $r^{\prime}<r$, this assertion is true. Apply the multi-linearlized Cayley-Hamilton theorem for $4 \times 4$-matrices $X_{1}, X_{2}, X_{3}, X_{4}$ to the case $X_{1}=$ $X(1)^{\alpha_{1}}, \quad X_{2}=X(2)^{\alpha_{2}}, \quad X(1)^{\alpha_{3}}, \quad X_{3}=X(2)^{\alpha_{4}}, X_{4}=X(1)^{\alpha_{5}} X(2)^{\alpha_{6}}$. Then by the inductive hypothesis we conclude the assertion. A similar argument shows that any invariant of the form

$$
\operatorname{Tr}\left(X(1)^{\alpha_{1}} X(2)^{\alpha_{2}} X(1)^{\alpha_{3}} X(2)^{\alpha_{4}} X(1)^{\alpha_{5}} X(2)^{\alpha_{8}}\right), \quad 1 \leqq \alpha_{1}, \alpha_{2}, \cdots, \alpha_{6} \leqq 3,
$$

is written as a polynomial in $T_{r}\left(X(1)^{\alpha_{1}} X(2)^{\alpha_{2}} X(1)^{\alpha_{3}} X(2)^{\alpha_{4}}\right), 0 \leqq \alpha_{1}, \cdots, \alpha_{4} \leqq 3$, $\operatorname{Tr}\left(X(1) X(2) X(1)^{2} X(2)^{2} X(1)^{3} X(2)^{3}\right), \quad \operatorname{Tr}\left(X(1) X(2) X(1) X(2)^{2} X(1) X(2)^{3}\right), \operatorname{Tr}(X(2)$. $\left.X(1) X(2) X(1)^{2} X(2) X(1)^{3}\right)$. The proposition is proved.

Set

$$
\begin{aligned}
& f_{1}=\operatorname{Tr}(X(1)), \quad f_{2}=\operatorname{Tr}\left(X(1)^{2}\right), \quad f_{3}=\operatorname{Tr}\left(X(1)^{3}\right), \quad f_{4}=\operatorname{Tr}\left(X(1)^{4}\right), \\
& f_{5}=\operatorname{Tr}(X(2)), \quad f_{6}=\operatorname{Tr}\left(X(2)^{2}\right), \quad f_{7}=\operatorname{Tr}\left(X(2)^{3}\right), \quad f_{8}=\operatorname{Tr}\left(X(2)^{4}\right), \\
& f_{9}=\operatorname{Tr}(X(1) X(2)), \quad f_{10}=\operatorname{Tr}\left(X(1)^{2} X(2)^{2}\right), \quad f_{11}=\operatorname{Tr}\left(X(1) X(2)^{2}\right), \\
& f_{12}=\operatorname{Tr}\left(X(1)^{2} X(2)\right), \quad f_{13}=\operatorname{Tr}\left(X(1) X(2)^{3}\right), \quad f_{14}=\operatorname{Tr}\left(X(1)^{3} X(2)\right), \\
& f_{15}=\operatorname{Tr}(X(1) X(2) X(1) X(2)), \quad f_{16}=\operatorname{Tr}\left(X(1) X(2)^{2} X(1) X(2)^{2}\right), \\
& f_{17}=\operatorname{Tr}\left(X(2) X(1)^{2} X(2) X(1)^{2}\right) .
\end{aligned}
$$

We denote by $C$ a subring of $C[X(1), X(2)]^{G L(4)}$ generated by $f_{1}, \cdots, f_{17}$.

Proposition 5.2. $f_{1}, \cdots, f_{17}$ is a homogeneous system of parameters o, the ring of invariants $C[X(1), X(2)]^{G L(4)}$.

Proof. Since the transcendence degree of the ring $C[X(1), X(2)]^{G L(4)}$ is 17, it is enough to show that, for $4 \times 4$-matrices $A_{1}$ and $A_{2}, f_{1}\left(A_{1}, A_{2}\right)=$ $\cdots=f_{17}\left(A_{1}, A_{2}\right)=0$ imply $\operatorname{Tr}\left(A_{i_{1}}, A_{i_{2}} \cdots A_{i_{k}}\right)=0, i_{1}, \cdots, i_{k}=1,2$ for any $k$.

Notice that $A_{1}^{4}=A_{2}^{4}=0$, since $f_{1}\left(A_{1}, A_{2}\right)=\cdots=f_{8}\left(A_{1}, A_{2}\right)=0$. Assume that $A_{1}^{3} \neq 0$. Then, by the substitution $A_{i} \rightarrow B A_{i} B^{-1}, B \in G L(4)$ and $i=$ 1,2 , we can assume that

$$
A_{1}=\left(\begin{array}{rrrr}
0 & 1 & & \\
& 0 & 1 & \\
& & 0 & 1 \\
& & & 0
\end{array}\right) \quad \text { and } \quad A_{2}=\left(\begin{array}{lllll}
a_{11} & a_{12} & a_{13} & a_{14} \\
a_{21} & a_{22} & a_{23} & a_{24} \\
a_{31} & a_{32} & a_{33} & a_{34} \\
a_{41} & a_{42} & a_{43} & a_{44}
\end{array}\right)
$$

It follows from the equations $\operatorname{Tr}\left(A_{1}^{2} A_{2}\right)=\operatorname{Tr}\left(A_{1}^{3} A_{2}\right)=0$ that $a_{41}=a_{31}$ $+a_{42}=0$ and the Cayley-Hamilton theorem shows that the equation $\operatorname{Tr}\left(A_{1}^{2} A_{2} A_{1}^{2} A_{2}\right)=0$ implies $\operatorname{Tr}\left(A_{1}^{2} A_{1} A_{2} A_{1} A_{2}\right)=0$. 
Since

$$
A_{1} A_{2}=\left(\begin{array}{llll}
a_{21} & a_{22} & a_{23} & a_{24} \\
a_{31} & a_{32} & a_{33} & a_{34} \\
0 & a_{42} & a_{43} & a_{44} \\
0 & 0 & 0 & 0
\end{array}\right)
$$

it follows from the equation $\operatorname{Tr}\left(A_{1}^{2} A_{1} A_{2} A_{1} A_{2}\right)=0$ that $a_{31} a_{42}=0$ and hence we have $a_{31}=a_{42}=0$. Then it follows from the relation $\operatorname{Tr}\left(A_{1} A_{2}\right)=a_{21}+$ $a_{32}+a_{43}=0$ that $\operatorname{Tr}\left(A_{1}^{2} A_{2}^{2}\right)=a_{21} a_{32}+a_{32} a_{43}=-a_{32}^{2}$ and we obtain $a_{32}=0$.

Since

$$
\begin{aligned}
\operatorname{Tr}\left(A_{1} A_{2} A_{1} A_{2}\right) & =\operatorname{Tr}\left(\left[\begin{array}{llll}
a_{21} & a_{22} & a_{23} & a_{24} \\
0 & 0 & a_{33} & a_{34} \\
0 & 0 & a_{43} & a_{44} \\
0 & 0 & 0 & 0
\end{array}\right)^{2}\right) \\
& =a_{21}^{2}+a_{43}^{2},
\end{aligned}
$$

$a_{21}=a_{43}=a_{32}=0$ and hence $A_{2}$ is a $4 \times 4$ upper triangular matrix with zero diagonal entries. Consequently we can conclude that $\operatorname{Tr}\left(A_{i_{1}}, A_{\iota_{2}} \ldots\right.$ $\left.A_{i_{k}}\right)=0,1 \leqq i_{1}, i_{2}, \cdots, i_{k} \leqq 2$ for any $k$. By the same argument, we obtain the same conclution if $A_{2}^{3} \neq 0$.

We next assume that $A_{1}^{3}=A_{2}^{3}=0$ and either $A_{1}^{2}$ or $A_{2}^{2}$ is not zero. Then we can take $A_{1}$ as

$$
A_{1}=\left(\begin{array}{llll}
0 & 1 & & \\
& 0 & 1 & \\
& & 0 & \\
& & & 0
\end{array}\right) \quad \text { or } \quad\left[\begin{array}{llll}
0 & & & \\
& 0 & 1 & \\
& & 0 & 1 \\
& & & 0
\end{array}\right),
$$

and divide into two cases:

Case 1.

$$
A_{1}=\left(\begin{array}{llll}
0 & 1 & & \\
& 0 & 1 & \\
& & 0 & \\
& & & 0
\end{array}\right], \quad A_{2}=\left(\begin{array}{llll}
a_{11} & a_{12} & a_{13} & a_{14} \\
a_{21} & a_{22} & a_{23} & a_{24} \\
a_{31} & a_{32} & a_{33} & a_{34} \\
a_{41} & a_{42} & a_{43} & a_{44}
\end{array}\right) \text {. }
$$

In this case, it follows from the equations $\operatorname{Tr}\left(A_{1}^{2} A_{2}\right)=0, \operatorname{Tr}\left(A_{1} A_{2} A_{1} A_{2}\right)=0$ and $\operatorname{Tr}\left(A_{1} A_{2}\right)=0$ that $a_{21}=a_{31}=a_{32}=0$. 
Therefore $A_{1} A_{2}$ and $A_{1}^{2} A_{2}$ are upper triangular matrices with zero diagonal entries. Similarly, replacing $A_{2}$ by $A_{2}^{2}$, we see that $A_{1} A_{2}^{2}$ and $A_{1}^{2} A_{2}^{2}$ are also upper triangular matrices with zero diagonal entries. This shows that $\operatorname{Tr}\left(A_{i_{1}} A_{i_{2}} \cdots A_{i_{k}}\right)=0,1 \leqq i_{1}, i_{2}, \cdots, i_{k} \leqq 2$ for any $k$.

Case 2.

$$
A_{:}=\left(\begin{array}{rrr}
0 & & \\
& 0 & 1 \\
& 0 & 1 \\
& & 0
\end{array}\right) \quad A_{2}=\left(\begin{array}{llll}
a_{11} & a_{12} & a_{13} & a_{14} \\
a_{21} & a_{22} & a_{23} & a_{24} \\
a_{31} & a_{32} & a_{33} & a_{34} \\
a_{41} & a_{42} & a_{43} & a_{44}
\end{array}\right) .
$$

In this case, by the equation $\operatorname{Tr}\left(A_{1}^{2} A_{2}\right)=0$, we have $a_{42}=0$.

Since

$$
A_{1} A_{2}=\left(\begin{array}{cccc}
0 & 0 & 0 & 0 \\
a_{31} & a_{32} & a_{33} & a_{34} \\
a_{41} & a_{42} & a_{43} & a_{44} \\
0 & 0 & 0 & 0
\end{array}\right)
$$

and $\operatorname{Tr}\left(A_{1} A_{2} A_{1} A_{2}\right)=0$, we have $a_{32}=a_{43}=0$. Then we find that $A_{1} A_{2} A_{1}$ $=a_{33} A_{1}^{2}$ and, replacing $A_{2}$ by $A_{2}^{2}, A_{1} A_{2}^{2} A_{1}=b A_{1}^{2}$. Here $b$ denotes the (3,3)entry of the matrix $A_{2}^{2}$.

Notice that, for any $4 \times 4$-matrix $X=\left(x_{i j}\right)$,

$$
A_{1}^{2} X=\left(\begin{array}{cccc}
0 & x_{32} & x_{33} & x_{34} \\
0 & 0 & 0 & 0 \\
0 & 0 & 0 & 0 \\
0 & 0 & 0 & 0
\end{array}\right) .
$$

Therefore we can conclude that $\operatorname{Tr}\left(A_{i 1} A_{i 2} \cdots A_{i k}\right)=0$ for any $k$.

If $A_{1}^{2}=A_{2}^{2}=0$, we have evidently $\operatorname{Tr}\left(A_{i 1} A_{i 2} \cdots A_{i k}\right)=0$. This completes the proof.

Proposition 5.2 shows that $C$ is a polynomial ring in 17 variables and $C[X(1), X(2)]^{G L(4)}$ is a free module over $C$.

\section{REFERENCES}

[1] E. Formanek, The center of the ring $3 \times 3$ generic matrices, Linear and Multilinear Algebra, 7 (1979), 203-212.

[2] - P. Halpin and W.-C. W. Li, The Poincaré series of the ring of $2 \times 2$ generic matrices, J. Algebra, 69 (1981), 105-112. 
[3] D. Hilbert, Über die vollen Invariantensysteme, Ges. Abh., II, 287-344, SpringerVerlag, 1970.

[4] M. Hochster and J. Roberts, Rings of invariants of reductive groups acting on regular rings are Cohen-Macaulay, Adv. in Math., 13 (1974), 115-175.

[5] C. Procesi, The invariant theory of $n \times n$ matrices, Adv. in Math., 19 (1976), 306-381.

[6] M. Rosenlict, A remark on quotient spaces, An. Acad. Brasil Ciênc., 35, (1963), $487-489$

[7] T. A. Springer, Invariant theory Springer Lecture note 585, 1977.

[8] H. Weyl, The classical groups, Princeton Univ. Press, Princeton, N. J., 1946.

[9] — Z Zur Darstellungstheorie und Invariantenabzählun gder projection, der Komplex und Drehungsgroppe, Ges. Abh., III, 1-25, Springer-Verlag, 1968.

Department of Mathematics

Faculty of Science

Nagoya University

Chikusa-ku, Nagoya, 464

Japan 